\title{
Model order reduction of parametric mechanical problems involving contacts
}

\author{
Kiran S. Kollepara **, José V. Aguado ${ }^{\dagger}$, Luisa Silva ${ }^{\dagger}$, Yves Le Guennec * and Stephanie \\ Colliou * \\ * Institut de Calcul Intensif \\ Ecole Centrale de Nantes \\ Rue de la Noë, 44321 Nantes Cedex 3, France \\ E-mail : kiran-sagar.kollepara.2@ec-nantes.fr, webpage : http://ici.ec-nantes.fr \\ $\dagger$ Institut de Calcul Intensif \\ Ecole Centrale de Nantes \\ Rue de la Noë, 44321 Nantes Cedex 3, France \\ webpage : http://ici.ec-nantes.fr \\ $\ddagger$ IRT Jules Verne \\ Chemin du Chaffault \\ 44340 Bouguenais, France \\ webpage : https://www.irt-jules-verne.fr
}

\begin{abstract}
In the context of manufacturing of engineering structures, unavoidable process variations can cause deviations in a manufactured structural part, resulting in "non-conformance" to the tolerance specifications. Prior to assembly, numerical simulations are often used to determine the effect of the non-conformances. Based on the analysis, parts with non-conformances are either accepted, rejected or reworked. However, the computational complexity of these numerical simulations delays the manufacturing schedule significantly. A real-time simulation tool, capable of evaluating the effect of non-conformances, is necessary to eliminate the bottleneck in the decision-making process.

To accelerate the simulation of structural assemblies, we propose to apply model order reduction methods on mechanical problems involving non-linear contact interfaces. Reduction of contact problems involve a special challenge of solving a non-linear inequality constrained minimization problem, including a non-negativity condition on the contact pressure. Current approaches use mixed reduced bases for displacement and contact pressure fields, an orthogonal basis for displacement and a non-negative basis for contact pressure [1,2].

The contact pressure field is not linearly separable in nature. Hence, treating the contact problem as a low rank problem can reduce accuracy of the solution. We propose to resolve the linear inseparability using over-complete dictionaries along with sparse decomposition methods. The usage of sparse decomposition methods is inspired from the sparse behaviour observed in existing reduced approach. Sparse decomposition methods include methods like LASSO, Orthogonal Matching Projection, etc [3]. Sparse methods will be adapted to take into account inequality constraints, which are a crucial part of contact mechanics problems.
\end{abstract}

\section{REFERENCES}

[1] A. Benaceur, A. Ern and V. Ehrlacher, A reduced basis method for parametrized variational inequalities applied to contact mechanics. Intl. J. for Numerical Methods in Engg., 121(6), 1170-1197, 2019

[2] M. Balajewicz, D. Amsallem, \& C. Farhat, Projection-based model reduction for contact problems. Intl. J. for Numerical Methods in Engineering, 106(8), 644-663, 2016

[3] J. Mairal, F. Bach, \& J. Ponce, Sparse modeling for image and vision processing. Foundations and Trends in Computer Graphics and Vision, 8(2-3), 85-283, 2014 